\title{
Advancing Transdisciplinary Translation for Prevention of High-Risk Behaviors: Introduction to the Special Issue
}

\author{
Diana H. Fishbein • Ty A. Ridenour \\ Published online: 12 April 2013 \\ (C) Society for Prevention Research 2013
}

\section{Scope of the Problem}

Estimates of the total overall costs of substance abuse in the United States, including productivity and health- and crimerelated costs exceed $\$ 600$ billion annually (National Drug Intelligence Center 2011). This includes approximately $\$ 181$ billion for illicit drugs, $\$ 193$ billion for tobacco, and $\$ 235$ billion for alcohol. In the USA, the root cause for $25 \%$ of the total deaths can be attributed to substance abuse (Kochanek et al. 2011). The most commonly used drug among American youth is alcohol. Alcohol, the most commonly used drug among American teenagers, kills youth 6.5 times more often than other drugs of abuse (Rehm et al. 2009). And in the larger population, a death toll of nearly 10,000 can be attributed to chronic alcohol abuse within a timeframe of 1 year. Drug consumption of all types generally begins during adolescence; by 13 years of age, over $30 \%$ of teens report having used at least one illicit substance (www.monitoringthefuture.org). As staggering as these numbers are, they do not fully describe the breadth of destructive public health and safety implications of substance abuse, such as family disintegration, loss of employment, failure in school, domestic violence, and child abuse, as well as the association of substance abuse with risky sex, HIV and other sexually transmitted diseases, heart disease, and cancer.

In response to the gravity of this issue, hundreds of millions of research dollars have been spent on preventive intervention programs aimed at curbing high-risk behaviors, such as substance abuse. At the National Institutes of Health (NIH) alone, \$1,674 million was spent in 2010 on substance abuse; more so than any other neurobehavioral disease. Despite this enormous amount of scholarly effort, however,

D. H. Fishbein $(\bowtie)$

RTI International, 5520 Research Park Drive, Suite 210, Baltimore, MD 21104, USA

e-mail: dfishbein@rti.org

T. A. Ridenour

University of Pittsburgh, Pittsburgh, PA, USA most prevention research on substance abuse has neither focused upon, nor sought to ameliorate generative and oftentimes malleable mechanisms (e.g., neurocognitive deficits, stress reactivity) in substance abuse and related risk behaviors. In effect, although some recipients of prevention programming engage positively and achieve long-term success across multiple domains of functioning, many others respond less favorably, showing a trajectory toward onset and escalation of behavioral maladjustments, drug use initiation and escalation, lack of intervention engagement, and persistent intervention resistance. This problem has led to calls by many (National Research Council and Institute of Medicine 2009; Sloboda and Bukoski 2003) for a multiple-systems transdisciplinary approach that incorporates perspectives from fields such as genetics, neuroscience, developmental epidemiology, contextual behavioral science, environmental sciences, and clinical practice. Such an approach is crucial if we are to significantly advance scientific knowledge and the development of successful interventions in this area. Translation of findings on etiological underpinnings of substance abuse and related behaviors will lead to a better understanding of the significant impact of the social and physical environment on neurogenetic systems in ways that will inform development of personalized prevention approaches.

\section{National Scientific Conference Sets the Stage}

To address these outstanding issues, a select group of 65 senior investigators, federal administrators and early career scientists attended a 2-day conference (April, 2012) funded by an NIH National Institute of Nursing Research grant (Dr. Diana Fishbein, PI), entitled "Advancing Transdisciplinary Translation for Prevention of High-Risk Behaviors." The goal was to develop an infrastructure and research agenda for developing a large-scale transdisciplinary program of research to translate existing knowledge into effective population-wide prevention of risk behaviors, including drug abuse. A diverse array of scientific capabilities and disciplines was represented 
to enhance the potential to move the field forward in significant and innovative ways. The meeting focused on increasing communication among scientists who are already investigating disparate aspects of high-risk behaviors but who have yet to achieve an integration of perspective and approach. To facilitate the transfer of information via identification of outstanding issues and cross-cutting collaborations, the agenda included ample discussion geared toward the generation of specific action steps designed to enable the field to accomplish these goals (see http://www.ttpr.org/ for proceedings and related materials). As the development of both this Special Issue and the conference agenda occurred simultaneously, they were reciprocally influential in helping to establish the parameters for this growing national initiative.

\section{The Need for a New More Encompassing Model}

There is pervasive (albeit not all inclusive) agreement that individual differences in risk for substance abuse can only be thoroughly understood by recognizing that human behavior is a reflection of genetic and neurobiological mechanisms that are sculpted and dynamically impacted by socioenvironmental factors (see Fig. 1). Interactions between these underlying mechanisms and exposures to a nurturing versus adverse environment bias developmental trajectories toward favorable or psychopathological outcomes, respectively. For example, there is evidence that certain genetic variants in dopaminergic systems may increase risk for substance abuse (Le Foll et al. 2009), possibly through alterations in decisionmaking ability, novelty seeking, and other cognitive and behavioral traits (Lusher et al. 2001; O'Sullivan et al. 2009). Additionally, environmental factors (such as stress differentially impacting individuals with and without these genetic variants) contribute to different outcomes (Brody et al. 2012; Caspi et al. 2003; Enoch 2011). Importantly, neural dysfunctions that often underlie behavioral problems like substance abuse, regardless of their origins, are often preventable and to some extent malleable. Targeted psychosocial-, educational-, and technological-based interventions may strengthen or compensate for cognitive and emotional regulatory processes (and their neural substrates) that often accompany and antedate substance abuse (Diamond and Lee 2011; Hermann and Parente 1996; Klingberg et al. 2005; Manchester et al. 1997; Riggs et al. 2006; Shaywitz et al. 2004). Such findings have direct implications for designing interventions to prevent substance abuse and, thus, have extraordinary potential significance for mental and public health policies.

To achieve an understanding of mechanisms of behavioral change, it is critical to incorporate basic science knowledge into developmental psychology and social science models, such as the broad research model illustrated in Fig. 1. The transfer of information from the basic sciences to the science/practice of prevention and back again, however, has been sluggish at best and nonexistent in many areas. As a result, there are few attempts to test comprehensive models of substance abuse informed by a range of relevant behavioral sciences. Moreover, few studies have focused on the functional moderators of intervention responsivity using measures shown to be sensitive and specific to the underlying generators of behavior. And even fewer studies have sought to identify mediators of intervention effects, such as focusing on generative, yet malleable, neurobiological systems. In general, channels of communication to increase familiarity with and appreciation for these seemingly divergent perspectives and methods among disciplines are poor and not well incentivized. Also, the basic and prevention sciences rarely address mechanistic issues in ways that are directly pertinent to one another. There is also a systematic failure of new scientific information to reach individuals, practitioners, communities, health care systems, and policymakers in a timely and interpretable manner to design or implement impactful programs (Dougherty and Conway 2008).

A program of research that utilizes transdisciplinary concepts and methods to achieve translational goals thus has potential to fill critical gaps in prevention science and practice. The complementarity of merging these methods and goals is exemplified in their definitions, largely but not exclusively proffered by NIH (Choi and Pak 2006). Transdisciplinary research requires synthesis of perspective and approach in terms of developing a common research question, sharing and borrowing methods, creating a common conceptual framework, and either learning each other's disciplinary language or creating a new common language. In essence, disciplines or capabilities should be not be compartmentalized, with investigators performing different aspects of the work or contributing a particular capability. Rather, there should be a true integration of thought.

Translational research has been defined as the process of applying discoveries generated during research in the laboratory and in preclinical studies to the development of trials and studies in humans [as well as the process of applying bedside observations to inform benchtop discoveries]. It is also research either directly aimed at enhancing the adoption of best practices in the community, or contributing to eventual applications at a later point along the "translational pathway." It should be noted, however, that the literature has differentially defined Type 2 with some emphasizing clinical trials, medical care practices, and the need for studies to employ "controlled environments." This Special Issue focuses instead on preventive intervention and policy development, implementation, evaluation, and refinement in a variety of settings (including community and population level) based on the needs in the field of substance abuse prevention. 


\section{Transdisciplinary Translational Prevention: Research Model}

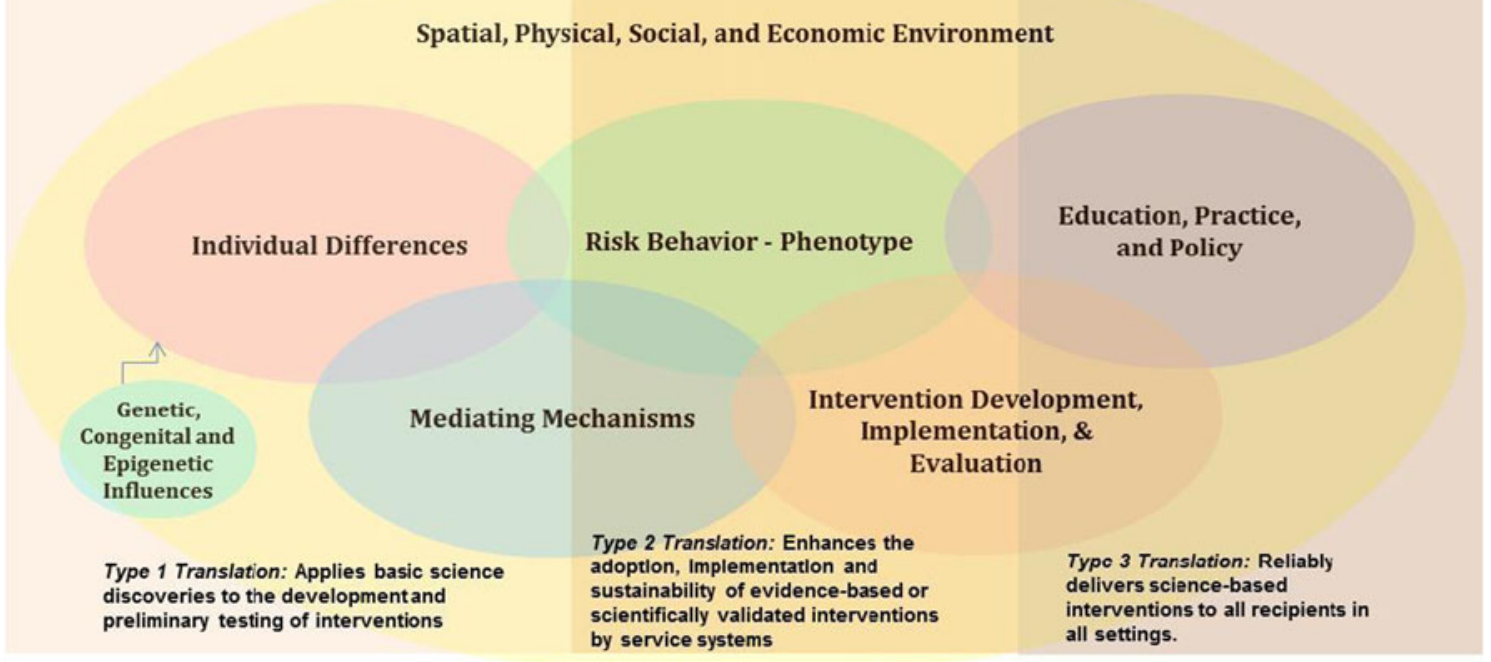

Fig. 1 Transdiscipinary translational prevention: Research model

\section{Premise for this Special Issue}

Significant gaps exist in our knowledge regarding malleable factors that moderate and mediate intervention outcomes due to the lack of communication among scientists investigating disparate aspects of the substance abuse issue. As a result, the transfer of information among disciplines has been slow and, more often than not, many years elapse after evidence accumulates to demonstrate a program's ineffectiveness before it is discontinued. Thus, although some recipients of prevention programming engage positively and achieve long-term success across multiple domains of functioning, many others respond less favorably, showing a trajectory toward onset and escalation of behavioral maladjustment, chronic relapses, lack of intervention engagement, and persistent intervention resistance. Because the factors underlying favorable and poor responses to extant interventions remain largely unknown, even "successful" programs, which at best have moderate efficacy, have limited clinical utility and appeal to end-users. One cumulative effect is that, in spite of considerable enthusiasm for prevention in communities, key players and their expert advisors lack the knowledge base and resources to implement impactful programs.

Identifying mechanisms of intervention outcomes will enhance understanding of what promotes or interferes with favorable outcomes; i.e., what works best for whom and why? Understanding such mechanisms will lead to improved, more targeted interventions for more persons and eventually sizably better outcomes in those who are less amenable to conventional approaches. Development, implementation, and refinement of the resultant science-based interventions in different populations and settings can then enhance etiology (backward translation) for more complete comprehension of underlying mechanisms of therapeutic outcomes for subgroups or individuals. The ultimate goal is that, through a transfer of knowledge from etiology to practice and back to etiology, public health strategies and policies will be increasingly responsive and effective, thereby exerting greater reductions in drug abuse and related risk behaviors.

\section{Topics of the Special Issue}

This Special Issue seeks to explicate the relevance, operational feasibility, and utility of a Transdisciplinary Translational Model to address issues pertinent to prevention of drug abuse (and its cognitive, emotional and behavioral antecedents) by demonstrating ways in which integrative techniques can elucidate mechanistic effects and moderators of intervention responsivity. This Special Issue and the NIH funded Conferences as well as a forthcoming monograph and website are all designed to promote cross-fertilization in the field of substance abuse prevention by stimulating interest in areas of expertise that cross the translational spectrum of behavioral research. Over time, it will be critical for this program of transdisciplinary translational research to address the aforementioned gaps in prevention research by identifying common ground and priorities, stimulating communication, sharing etiological findings, applying multilevel methodologies to 
analyzing integrated datasets, and determining ways in which collaborative investigations can lead to a better understanding of mechanisms of behavioral change. Ultimately, such programs of research will inform prevention practice and policy, potentially resulting in extraordinary cost savings and improved quality of life.

To invigorate transdisciplinary translational prevention science focused on substance abuse, the first three sections of this Special Issue highlight significant strides recently made toward identifying factors that moderate and mediate intervention outcomes. The first section presents innovative reanalyses of datasets from existing, traditional prevention clinical trials to determine how underlying mechanisms correlate and interact to promote or interfere with response to preventive interventions. Liu et al. (2013) demonstrate how efficacy research can inform developmental science as well as the potential for untargeted long-term benefits of prevention consequent to altering developmental trajectories. The following two articles address the role of executive cognitive functioning (ECF) in substance abuse. Pokhrel et al. (2013) present findings that support the notion that ECF subserves self-regulation of behavior and, as a result, directly influences risk for substance abuse. This information may be important in designing or refining school-based programs. Pentz and Riggs (2013) test the role(s) that ECF may play, not only in substance use etiology, but also in propensity for exercising and obesity. Both articles allude to the potential for interventions that more directly target these processes to exert a preventive effect (Pokhrel et al. 2013).

The second section of the Special Issue focuses specifically on integrating neuroscience into prevention research. A common goal of these papers is to equip prevention researchers with terminology, references, success examples and resources to examine the neurobiological underpinnings of not only the etiology of substance abuse but also the mechanisms of intervention efficacy. Whitten (2013) provides an instructive overview of neuroimaging techniques and their import to research that seeks to elucidate mechanisms in cognitive, emotional and behavioral antecedents and underpinnings of substance use. She highlights specific ways in which neuroimaging may be used to inform both prevention research and the development of more effective interventions. Other contributors have taken a developmental approach by differentiating between periods of adolescence (Eldreth et al. 2013) and young childhood (Bruce et al. 2013). Certain neural systems that are essential to resilient development are outlined within the context of prevention research and practice, including the hypothalamicpituitary-adrenocortical system (Bruce et al. 2013) and the interacting influences of self-regulation, reward reactivity, and emotion (Eldreth et al. 2013).

The third segment of the Special Issue presents analytic approaches that are nontraditional in prevention science but offer high potential to unveil developmental influences that facilitate or even result from intervention including systems science methods (Clark et al. 2013; Hassmiller Lich et al. 2013; Prado et al. 2013; Ridenour et al. 2013) and withinperson idiographic processes (Ridenour et al. 2013). Ridenour et al. (2013) explore the use of intensive withinperson lines of research to understand idiographic processes related to development and differential intervention responses. Hassmiller Lich et al. (2013) discuss application of analytic approaches with potential to capture a "cells to society" systems perspective; in particular, a systems sciences approach to problem solving and methods equipped to handle complex relationships and their evolution over time. Prado et al. (2013) investigate the role of subtypes of family characteristics in differential intervention responsivity. Neuroimaging (MRI) methods are discussed by Clark et al. (2013) with respect to the ability to identify structural and functional characteristics that may predict substance use disorder risks and effects, as well as to guide efforts to identify preventive intervention targets and outcome indicators. These methods were highlighted in recognition that multiple theoretical and methodological levels (i.e., from neurological to social and environmental) need to be understood in an effective and scientifically sound manner. In all cases, authors offered concrete ideas about how their work could translate into intervention services to advance conventional prevention strategies.

Completing the Special Issue, the fourth section deals with ethical questions that arise during any discussions of applying science to the early detection and intervention of youthful risk behaviors. Fisher and Harrington McCarthy (2013) address ethical issues that prevention scientists and practitioners face, particularly when investigating novel topics, using genetic research to illustrate the issues.

Articles included in this Special Issue illustrate innovative approaches to discovery using transdisciplinary translational perspectives and methods that are highly informative to prevention science and practice. These approaches fit not only the needs of the field but also research funders' priorities. The ultimate beneficiaries of expanding the scope of prevention science will be recipients of the resultant mechanism-driven, evidence-based interventions.

\section{References}

Brody, G. H., Chen, Y. F., Yu, T., Beach, S. R., Kogan, S. M., Simons, R. L., \& Philibert, R. A. (2012). Life stress, the dopamine receptor gene, and emerging adult drug use trajectories: A longitudinal, multilevel, mediated moderation analysis. Developmental Psychopathology, 24, 941-951.

Bruce, J., Gunnar, M. R., Pears, K. C., \& Fisher, P. A. (2013). Early adverse care, stress neurobiology, and prevention science: Lessons learned. Prevention Science. doi:10.1007/s11121-012-0354-6.

Caspi, A., Sugden, K., Moffitt, T. E., Taylor, A., Craig, I. W., Harrington, H., \& Poulton, R. (2003). Influence of life stress on depression: 
Moderation by a polymorphism in the 5-HTT gene. Science, 301, 386-389.

Choi, B. C., \& Pak, A. W. (2006). Multidisciplinarity, interdisciplinarity and transdisciplinarity in health research, services, education and policy: 1. Definitions, objectives, and evidence of effectiveness. Clinical \& Investigative Medicine, 29, 351364.

Clark, D. B., Chung, T., Pajtek, S., Zhai, Z., Long, E., \& Hasler, B. (2013). Neuroimaging methods for adolescent substance use disorder prevention science. Prevention Science. doi:10.1007/s11121-012-0323-0.

Diamond, A., \& Lee, K. (2011). Interventions shown to aid executive function development in children 4 to 12 years old. Science, 333, 959-964.

Dougherty, D., \& Conway, P. H. (2008). The "3T's" road map to transform US health care: The "how" of high-quality care. Journal of the American Medical Association, 299, 2319-2321.

Eldreth, D., Hardin, M. G., Pavletic, N., \& Ernst, M. (2013). Adolescent transformations of behavioral and neural processes as potential targets for prevention. Prevention Science. doi:10.1007/s11121012-0322-1.

Enoch, M. A. (2011). The role of early life stress as a predictor for alcohol and drug dependence. Psychopharmacology, 214, 17-31.

Fisher, C. B., \& Harrington McCarthy, E. L. (2013). Ethics in prevention science involving genetic testing. Prevention Science. doi:10.1007/s11121-012-0318-x.

Hassmiller Lich, K., Ginexi, E. M.., Osgood, N. D., \& Mabry, P. L. (2013). A call to address complexity in prevention science research. Prevention Science. doi:10.1007/s11121-012-0285-2.

Hermann, D., \& Parente, R. (1996). Retraining cognition: Techniques and application. Baltimore, MD: Aspen.

Klingberg, T., Fernell, E., Olesen, P. J., Johnson, M., Gustafsson, P., Dahlström, K., \& Westerberg, H. (2005). Computerized training of working memory in children with ADHD: A randomized, controlled trial. Journal of the American Academy of Child and Adolescent Psychiatry, 44, 177-186.

Kochanek, K.D., Jiaquan Xu, M.A., Murphy, S.L., Minino, A.M., \& Kung, H.-C. (2011). Deaths: Final data for 2009, Division of Vital Statistics (Atlanta, GA: Centers for Disease Control), 60, Dec. 29.

Le Foll, B., Gallo, A., Le Strat, Y., Lu, L., \& Gorwood, P. (2009). Genetics of dopamine receptors and drug addiction: A comprehensive review. Behavioral Pharmacology, 20, 1-17.

Liu, W., Lynne-Landsman, S. D., Petras, H., Masyn, K., \& Ialongo, N. (2013). The evaluation of two first-grade preventive interventions on childhood aggression and adolescent marijuana use: A latent transition longitudinal mixture model. Prevention Science. doi:10.1007/s11121-013-0375-9.

Lusher, J. M., Chandler, C., \& Ball, D. (2001). Dopamine D4 receptor gene (DRD4) is associated with novelty seeking (NS) and substance abuse: The saga continues.... Molecular Psychiatry, 6, 497-499.
Manchester, D., Hodgkinson, A., \& Casey, T. (1997). Prolonged, severe behavioral disturbance following traumatic brain injury: What can be done? Brain Injury, 11, 605-617.

National Drug Intelligence Center. (2011). The economic impact of illicit drug use on American society. Washington, DC: United States Department of Justice. Available at: http://www.justice.gov/archive/ ndic/pubs44/44731/44731p.pdf.

National Research Council and Institute of Medicine. (2009). Preventing mental, emotional and behavioral disorders among young people: progress and possibilities. Washington, DC: National Academies Press.

O'Sullivan, S. S., Evans, A. H., \& Lees, A. J. (2009). Dopamine dysregulation syndrome: An overview of its epidemiology, mechanisms and management. CNS Drugs, 23, 157-170.

Pentz, M. A., \& Riggs, N. R. (2013). Longitudinal relationships of executive cognitive function and parent influence to child substance use and physical activity. Prevention Science. doi:10.1007/ s11121-012-0312-3.

Pokhrel, P., Herzog, T. A., Black, D., Zamin, A., Riggs, N. R., \& Sussman, S. (2013) Adolescent neurocognitive development, selfregulation, and school-based drug use prevention. Prevention Science. doi:10.1007/s11121-012-0345-7.

Prado, G., Huang, S., Cordova, D., Malcolm, S., Estrada, Y., Cano, N., et al (2013). Ecodevelopmental and intrapersonal moderators of a family based preventive intervention for Hispanic youth: A latent profile analysis. Prevention Science. doi:10.1007/s11121-012-0326-x.

Rehm, J., Mathers, C., Popova, S., Thavorncharoensap, M., Teerawattananon, Y., \& Patra, J. (2009). Global burden of disease and injury and economic cost attributable to alcohol use and alcoholuse disorders. Lancet, 373, 2223-2233.

Ridenour, T. A., Pineo, T. Z., Maldonado-Molina, M. M., \& Hassmiller Lich, K. (2013). Toward rigorous idiographic research in prevention science: Comparison between three analytic strategies for testing preventive intervention in very small samples. Prevention Science. doi:10.1007/s11121-012-0311-4.

Riggs, N. R., Greenberg, M. T., Kusché, C. A., \& Pentz, M. A. (2006). The mediational role of neurocognition in the behavioral outcomes of a social-emotional prevention program in elementary school students: Effects of the PATHS Curriculum. Prevention Science, 7, 91-102.

Shaywitz, B. A., Shaywitz, S. E., Blachman, B. A., Pugh, K. R., Fulbright, R. K., Skudlarski, P., Mencl, W. E., Constable, R. T., Holahan, J. M., Marchione, K. E., Fletcher, J. M., Lyon, G. R., \& Gore, J. C. (2004). Development of left occipitotemporal systems for skilled reading in children after a phonologically-based intervention. Biological Psychiatry, 55, 926-933.

Sloboda, Z., \& Bukoski, W. J. (Eds.). (2003). Handbook of drug abuse prevention: theory, science, and practice. New York: Kluwer.

Whitten, L. A. (2013). Translational neuroscience and potential contributions of functional magnetic resonance imaging (fMRI) to the prevention of substance misuse and antisocial behavior. Prevention Science. doi:10.1007/s11121-012-0341-y. 